\title{
Monitoring of Superparamagnetic Particle Sizes in the Langevin Law Regime
}

\author{
Daniel Henrard $\left(D,{ }^{1}\right.$ Quoc Lam Vuong, ${ }^{1}$ Sébastien Delangre, ${ }^{1}$ Xavier Valentini, \\ Denis Nonclercq $\mathbb{D}^{2},{ }^{2}$ Maurice François Gonon, ${ }^{3}$ and Yves Gossuin $\mathbb{D i D}^{1}$ \\ ${ }^{1}$ Biomedical Physics Unit, Materials Institute, University of Mons, Place du Parc 20, 7000 Mons, Belgium \\ ${ }^{2}$ Histology Unit, University of Mons, Place du Parc 20, 7000 Mons, Belgium \\ ${ }^{3}$ Department of Materials Science, University of Mons, Place du Parc 20, 7000 Mons, Belgium
}

Correspondence should be addressed to Yves Gossuin; yves.gossuin@umons.ac.be

Received 8 May 2019; Accepted 17 September 2019; Published 19 November 2019

Academic Editor: Ovidiu Ersen

Copyright ( 2019 Daniel Henrard et al. This is an open access article distributed under the Creative Commons Attribution License, which permits unrestricted use, distribution, and reproduction in any medium, provided the original work is properly cited.

\begin{abstract}
In this work, aqueous solutions of magnetite nanoparticles (NPs) are studied. Magnetite NPs are very useful in biomedicine for magnetic resonance imaging (MRI), for drug delivery therapy, and also for hyperthermia. In order to predict the NP efficiency in these applications, it is crucial to accurately characterize their size distribution and their magnetization. Magnetometry, through the dependence of NP magnetization on the magnetic induction (MB curve), can provide interesting information on these physical properties. In this work, the extraction of the NP size distribution and magnetization from experimental MB curves of aqueous solutions of magnetite NPs is discussed. The results are compared to TEM and XRD characterizations. It is shown that an expression taking into account the size distribution better fits the results than the commonly used simple Langevin function. The size distributions obtained by magnetometry seem comparable to those obtained by TEM measurements. However, a closer look at the results shows some nonnegligible discrepancies: the size distributions obtained by magnetometry vary with the temperature and are closer to the TEM ones at room temperature. Our study suggests that it could be explained by the nonnegligible anisotropy energy of the NPs at low temperature and the lack of NP Brownian rotation below the freezing point of water. This demonstrates that care must be taken when interpreting the results obtained by magnetometry of magnetite NPs: only the size and size distribution obtained at room temperature should be used.
\end{abstract}

\section{Introduction}

1.1. Applications and Importance of NP Characterization. Magnetite nanoparticles (NPs) are very useful in biomedicine [1]. They find their applications as contrast agents in magnetic resonance imaging (MRI), for drug delivery therapy and also for hyperthermia. Each application requires NPs of specific size: in MRI, for example, large NPs $(60-150 \mathrm{~nm})$ are used for liver or spleen imaging because they are immediately captured by these organs, while small NPs $(<40 \mathrm{~nm})$ are used for MR angiography or lymph node imaging because they stay longer in the bloodstream [2]. For drug delivery and hyperthermia, the NPs have to be small enough so that a longer lifetime in the bloodstream allows them to reach the targeted organs. Developing reliable methods to know the NP size is thus crucial.
Furthermore, the NP size is one of the key parameters that drives the efficiency in each application. In MRI, for example, the NP "contrast agent effect" arises from their ability to locally decrease proton relaxation times [3]. Relaxation is the process by which the proton magnetic moments come back to equilibrium after a radiofrequency perturbation. Due to the presence of magnetic NPs, relaxation times are shortened and the image contrast is enhanced in regions where they are concentrated [3]. However, an optimum NP size exists to achieve this, from $30 \mathrm{~nm}$ to $400 \mathrm{~nm}$ depending on the type of magnetic particles [4].

In drug delivery therapy and hyperthermia, NPs are excited by a high-frequency alternating magnetic field [511]: it locally rises temperature in targeted organs which causes tumor cell destruction by thermally activated drug release $[7,9-11]$ and/or by thermal shock inducing apoptosis 
$[6-8,10,11]$. The heating is dependent on the hysteresis loop area which is strongly linked to NP size distribution. There also exists an optimum size to maximize this heating $[9,12$, 13]. In drug delivery therapy, targeting is usually carried out using antibodies or $\mathrm{pH}$ changes in targeted organs but can also be done using magnetic NP carriers guided magnetically $[10,14,15]$.

NP magnetization also plays a key role on the efficiency in biomedical applications. The larger the magnetization is, the better it is: the NP efficiency as contrast agent depends on the dipolar magnetic field they produce which will be larger for larger magnetization. Similarly, heating in drug delivery or hyperthermia technique is directly proportional to magnetization since a larger magnetization also increases the hysteresis loop area. That is why magnetite NPs are commonly chosen for these applications: if they are small enough (typically when their radius is smaller than $100 \mathrm{~nm}$ ), magnetite NPs are composed of only one single Weiss domain and then have a "super" magnetic moment $\mu$.

1.2. Superparamagnetism and Langevin Law. This type of magnetism is not only called superparamagnetism because of this large magnetic moment but also because only magnetic energy $\mu B$ and thermal energy $k_{B} T$ are taken into account like in paramagnetism: global sample magnetization of a superparamagnetic sample can be calculated with the Boltzmann statistics. When only these energy terms are taken into account, the magnetization is proportional to the Langevin function

$$
L(\xi)=\operatorname{coth}(\xi)-\frac{1}{\xi},
$$

where $\xi=\mu B / k_{B} T, k_{B}$ is the Boltzmann constant, $T$ the temperature, $\mu$ the NP magnetic moment, and $B$ the magnetic induction. The Langevin function, and thus magnetization, is equal to zero at zero magnetic induction which is also a characteristic of paramagnetism. On the opposite, it saturates quickly to 1 when magnetic induction increases which is typical of superparamagnetism.

When the Langevin function is used, it is important to keep in mind that anisotropy energy is not taken into account. Anisotropy energy $K_{A} V$, where $K_{A}$ is the material anisotropy constant and $V$ the NP volume, is usually introduced to reflect the presence of easy NP axes of magnetization while others are hard axes of magnetization. Since NPs are very small, anisotropy energy is very low: the NP magnetic moment can overcome the anisotropy energy barrier $\Delta E_{A}$ thanks to thermal energy and jump from one easy direction to another. The characteristic time between two jumps is called the "Néel relaxation time":

$$
\tau_{N}=\tau_{0} e^{\Delta E_{A} / k_{B} T}
$$

where $\tau_{0}$ is the characteristic time of magnetite which is in the $10^{-9}-10^{-13} s$ order of magnitude and $\Delta E_{A}=K_{A} V$ in the simplest case where the NP is characterized by only one anisotropy axis. This phenomenon is called Néel relaxation and is responsible for the zero global sample magnetization at zero magnetic induction and at room temperature in powder samples.

In unfrozen aqueous solutions, NPs are free to rotate so that they can change the orientation of their anisotropy axis to the magnetic field and minimize the magnetic energies. This second relaxation is called "Brown relaxation" and its characteristic time is

$$
\tau_{B}=\frac{3 \eta V_{H}}{k_{B} T}
$$

where $\eta$ is the liquid water viscosity and $V_{H}$ the hydrodynamic NP volume (magnetic core + coating). If $T>273.15$ $\mathrm{K}$, both relaxation mechanisms are present and the resultant relaxation characteristic time $\tau$ is

$$
\frac{1}{\tau}=\frac{1}{\tau_{N}}+\frac{1}{\tau_{B}} .
$$

Néel relaxation is dominant for small NPs $(r<9 \mathrm{~nm})$ when $\tau_{N}<\tau_{B}$, and Brown relaxation in liquid water is dominant for larger NPs $(r>9 \mathrm{~nm})$ when $\tau_{N}>\tau_{B}$ [12]. The measurement characteristic time for a vibrating sample magnetometer is $\tau_{x p}=0.1-10$ s. $\tau_{x p}>>\tau_{B}$ (at least 10 times larger) is verified as long as the NP hydrodynamic radius $r_{H}<115 \mathrm{~nm}$. Brown relaxation is thus sufficient: NPs are always free to rotate during measurement. Consequently, the average magnetic moment, given by the Langevin function, is measured.

For lower temperatures $(T<273.15 \mathrm{~K})$, i.e., frozen water, only Néel relaxation occurs. The NP magnetic moment is blocked in one energy minimum if $\tau_{x p}<\tau_{N}$. On the opposite, it can switch if $\tau_{x p}>\tau_{N}$ : consequently, the average magnetic moment, which is given by the Langevin function, is measured. At a specific temperature $T$, the condition $\tau_{x p}>\tau_{N}$ gives a condition on the radius:

$$
r<\sqrt[3]{\frac{3 k_{B} T \ln \left(\tau_{x p} / \tau_{0}\right)}{4 \pi K_{A}}}=r_{B} .
$$

This means that, at a specific temperature $T$, all NPs larger than the blocking radius $r_{B}$ have their magnetic moment blocked. So, these NPs can contribute to a nonzero magnetization at zero magnetic induction if they were magnetized before. Considering $\tau_{0}=10 s$ and $\tau_{x p}=10^{-13} s$ as given before, the maximum value for $\ln \left(\tau_{x p} / \tau_{0}\right)$ is 32 . On the opposite, considering $\tau_{0}=0.1 s$ and $\tau_{x p}=10^{-9} s$, the minimum value for $\ln \left(\tau_{x p} / \tau_{0}\right)$ is 18. In this work, 25 was arbitrarily chosen as the mean value. If one considers $K_{A}=15000 \mathrm{~J} / \mathrm{m}^{3}[10,16-19]$, the blocking radius $r_{B}$ is equal to $12 \mathrm{~nm}$ at $300 \mathrm{~K}, 8 \mathrm{~nm}$ at $100 \mathrm{~K}$, and $2 \mathrm{~nm}$ at $2 \mathrm{~K}$.

1.3. Characterization Issues. NP characterization is thus essential. However, measuring NP size is not straightforward. Depending on the technique, only the magnetic core size is observed or the entire entity (magnetic core + coating). Transmission electron microscopy (TEM) or X-ray 
diffraction (XRD) allows the determination of the magnetic core size. That is also the case with magnetometry which studies the evolution of magnetization with the magnetic induction and temperature. On the opposite, dynamic light scattering (DLS) or NMR relaxometry which studies relaxation times at varying magnetic fields provides the hydrodynamic size. Care should thus be taken when comparing values obtained with different techniques.

NPs are generally not monodispersed, i.e., they are characterized by a size distribution. Since a size distribution is always present, the different techniques will yield different mean NP sizes. Indeed, for each technique, the NP contribution is different [20]: in magnetometry, the signal is proportional to $r^{3}$ while in relaxometry it is proportional to $r^{5}$. In both cases, large NP weighting is higher than for small ones. As a result, the mean NP size is artificially overestimated. This is even more marked in relaxometry. One way to solve this issue (at least partly) is to take into account the size distribution in the experimental curve adjustment. This solution can be rather easily implemented in magnetometry as shown in many papers before [16, 21-27].

Indeed, it has been shown that NP size distribution can be estimated by adjusting magnetization in function of the magnetic induction (MB curves) with a relatively good precision [16, 22, 23, 25-27]. If many papers have studied single $\mathrm{MB}$ curves (at one single temperature), none of them has evaluated the NP size via a set of MB curves obtained at different temperatures. Most of the studies performed this estimation at room temperature [16, 23, 25-27]. However, the sample was cooled down before the measurement in Vaishnava et al. [22]. Does temperature influence the NP size evaluation via $\mathrm{MB}$ experiments? This question will be studied in this paper. TEM results will be used as reference.

\section{Materials and Methods}

2.1. Solutions. Four aqueous solutions (S1, S2, S3, and S4) containing magnetite/maghemite $\left(\mathrm{Fe}_{3} \mathrm{O}_{4} / \mathrm{Fe}_{2} \mathrm{O}_{3}\right)$ NPs were ordered from Ferropharm Gmbh (Teltow, Germany). NPs are coated with sodium citrate to avoid clustering. According to Ferropharm Gmbh, each solution has an iron concentration of $500 \mathrm{mM}$.

2.2. XRD. X-ray diffraction (XRD) was performed in the Department of Materials Science of UMONS with a Bruker D5000 (Billerica, MA, USA) on lyophilized powders of the solutions, using an anticathode made of copper and a wavelength $\lambda=0.15406 \mathrm{~nm}\left(K_{\alpha}\right.$ spectral line). XRD profiles were acquired at room temperature with a $9.6 \mathrm{~s}$ time step and a $0.02^{\circ}$ angle step from $25^{\circ}$ to $70^{\circ}$. Peaks overlapping confirmed that solutions contained a magnetite/maghemite mixture even if maghemite peaks were predominant.

2.3. TEM. Each solution was diluted to obtain an iron concentration of $0.1-0.5 \mathrm{mM}$. This new solution was sprayed on gold grids covered by a formvar film from Agar Scientific Ltd. (Stansted, UK). Vaporization avoids cluster formation during evaporation. After $24 \mathrm{~h}$ drying, transmission electron microscopy images were carried out in the Histology Unit of UMONS with a LEO 906E microscope (Zeiss, Germany) operating at $80 \mathrm{kV}$. ImageJ software was used to size manually at least $200 \mathrm{NPs}$ for each sample. Finally, a histogram with 21 to 27 bins was created and adjusted with a lognormal size distribution:

$$
f_{\log }\left(r, r_{0}, \sigma\right)=\frac{1}{r \sigma \sqrt{2 \pi}} e^{-\left(\ln r / r_{0}\right)^{2} / 2 \sigma^{2}},
$$

where $r_{0}$ is the median radius and $\sigma$ the radius natural logarithm standard deviation. TEM results are given in Table 1. Gaussian distribution is sometimes used in the literature but this distribution is less usual. Lognormal distribution allows to represent an asymmetric distribution in the NP sizes, with less small NPs and more large NPs, and was previously introduced by Chantrell et al. [21] and O'Grady and Bradbury [28]. For our samples, lognormal distribution works slightly better than Gaussian distribution to adjust the TEM histograms: average $R_{\text {adj }}^{2}$ for all samples is equal to 0.9598 for lognormal distribution fitting compared to 0.9375 for Gaussian distribution fitting.

2.4. Magnetometer. For the magnetometric experiments, a vibrating sample magnetometer (VSM) from Cryogenic Ltd. (London, UK) was used. Sample temperature ranges from $2 \mathrm{~K}$ to $315 \mathrm{~K}$, and the maximum magnetic induction is $5 \mathrm{~T}$. The theoretical sensitivity is $10^{-6} \mathrm{emu}$. For the first step of calibration, sample height is fixed to maximize the signal detected in the two detection coils. Then, amplitude signal is tuned to the signal of an Yttrium-iron reference ball from National Institute of Standards \& Technology (Gaithersburg, $\mathrm{MD}$, USA). Its mass is $2.836 \mathrm{mg}$, and its magnetic moment is equal to $78.3 \cdot 10^{-3} \mathrm{emu}$ at $0.5 \mathrm{~T}$ and $298 \mathrm{~K}$.

2.5. Experiments. All samples were prepared under a fume hood to avoid contamination by impurities. Sample vials in polyethylene from Sigma-Aldrich (Saint Louis, MO, USA) are filled with $50 \mu \mathrm{l}$ of solution. MB curves have been performed from $5 \mathrm{~T}$ to $-5 \mathrm{~T}$ at $100 \mathrm{~K}, 200 \mathrm{~K}, 275 \mathrm{~K}, 300 \mathrm{~K}$, and $315 \mathrm{~K}$. Samples were cooled from room temperature under zero magnetic field at around $1 \mathrm{~K} / \mathrm{min}$.

2.6. Data Processing. No paramagnetic signal has been observed on any of the samples as it is sometimes the case for such NPs because of surface disorder [29-31]. Only the decreasing diamagnetic signal coming from water and from the sample vial was present at high field and was in the order of magnitude of expected water contribution. This diamagnetic signal was subtracted by considering that as soon as $B \mid>2.5 T$, the observed small signal decrease was only due to diamagnetism. All the points corresponding to $B>2.5 T$ and $B<-2.5 T$ were simultaneously adjusted with two linear expressions $(p B+c s t$ and $p B-c s t$, respectively). For each $\mathrm{MB}$ experiment, the magnetic moment was corrected using $\mu_{\text {corr }}=\mu_{\text {mes }}-p B$.

Iron concentration of each solution was determined by Inductively Coupled Plasma-Atomic Emission Spectroscopy (ICP-AES) after microwave digestion in $8 \mathrm{ml}$ nitric acid 
TABLE 1: Saturation magnetizations measured in MB curves (first part of the table). Results of MB curve fitting obtained with lognormal size distribution fitting (LDF) and simple Langevin fitting (SLF) (i.e., no size distribution); size distribution from TEM.

\begin{tabular}{|c|c|c|c|c|c|c|}
\hline & $\mathrm{MB}$ & & S1 & S2 & S3 & S4 \\
\hline & $100 \mathrm{~K}$ & $M_{s}(\mathrm{emu} / \mathrm{g} \mathrm{Fe})$ & $114.1(3.4)$ & $114.8(3.4)$ & $103.9(3.1)$ & $104.4(3.1)$ \\
\hline & $200 \mathrm{~K}$ & $M_{s}(\mathrm{emu} / \mathrm{g} \mathrm{Fe})$ & $104.8(3.1)$ & $107.3(3.2)$ & $98.0(2.9)$ & $97.3(2.9)$ \\
\hline & $275 \mathrm{~K}$ & $M_{s}(\mathrm{emu} / \mathrm{g} \mathrm{Fe})$ & $99.4(3.0)$ & $100.0(3.0)$ & $92.2(2.8)$ & $89.3(2.7)$ \\
\hline & $300 \mathrm{~K}$ & $M_{s}(\mathrm{emu} / \mathrm{g} \mathrm{Fe})$ & $95.1(2.9)$ & $96.4(2.9)$ & $89.0(2.7)$ & $87.1(2.6)$ \\
\hline & $315 \mathrm{~K}$ & $M_{s}(\mathrm{emu} / \mathrm{g} \mathrm{Fe})$ & $93.3(2.8)$ & $94.4(2.8)$ & $86.9(2.6)$ & $85.0(2.5)$ \\
\hline \multirow{10}{*}{ LDF } & \multirow{2}{*}{$100 \mathrm{~K}$} & $r_{0}(\mathrm{~nm})$ & $2.05(7)$ & $2.67(7)$ & $2.27(7)$ & $1.86(5)$ \\
\hline & & $\sigma$ & $0.4176(43)$ & $0.2956(18)$ & $0.4182(38)$ & $0.4704(27)$ \\
\hline & \multirow{2}{*}{$200 \mathrm{~K}$} & $r_{0}(\mathrm{~nm})$ & $2.95(8)$ & $3.22(8)$ & $2.95(8)$ & $2.45(7)$ \\
\hline & & $\sigma$ & $0.2910(31)$ & $0.2132(25)$ & $0.3859(30)$ & $0.4184(29)$ \\
\hline & \multirow{2}{*}{$275 \mathrm{~K}$} & $r_{0}(\mathrm{~nm})$ & $3.41(9)$ & $3.46(9)$ & $3.88(11)$ & $2.92(9)$ \\
\hline & & $\sigma$ & $0.2084(30)$ & $0.1684(35)$ & $0.3049(27)$ & $0.3683(34)$ \\
\hline & \multirow{2}{*}{$300 \mathrm{~K}$} & $r_{0}(\mathrm{~nm})$ & $3.52(10)$ & $3.53(9)$ & $3.96(11)$ & $2.99(9)$ \\
\hline & & $\sigma$ & $0.2057(35)$ & $0.1572(39)$ & $0.2971(26)$ & $0.3640(37)$ \\
\hline & \multirow{2}{*}{$315 \mathrm{~K}$} & $r_{0}(\mathrm{~nm})$ & $3.59(10)$ & $3.60(10)$ & $4.08(11)$ & $3.07(9)$ \\
\hline & & $\sigma$ & $0.1803(41)$ & $0.1443(49)$ & $0.2859(24)$ & $0.3488(35)$ \\
\hline \multirow{7}{*}{ SLF } & $100 \mathrm{~K}$ & $r_{0}(\mathrm{~nm})$ & $3.30(9)$ & $3.37(9)$ & $3.62(10)$ & $3.42(10)$ \\
\hline & $200 \mathrm{~K}$ & $r_{0}(\mathrm{~nm})$ & $3.77(10)$ & $3.68(9)$ & $4.42(12)$ & $4.04(11)$ \\
\hline & $275 \mathrm{~K}$ & $r_{0}(\mathrm{~nm})$ & $3.90(10)$ & $3.78(9)$ & $5.00(13)$ & $4.35(11)$ \\
\hline & $300 \mathrm{~K}$ & $r_{0}(\mathrm{~nm})$ & $3.94(10)$ & $3.82(9)$ & $5.05(13)$ & $4.42(11)$ \\
\hline & $315 \mathrm{~K}$ & $r_{0}(\mathrm{~nm})$ & $3.97(10)$ & $3.85(9)$ & $5.12(13)$ & $4.43(11)$ \\
\hline & \multirow{2}{*}{ TEM } & $r_{0}(\mathrm{~nm})$ & $3.170(26)$ & $3.228(30)$ & $3.622(43)$ & $3.32(5)$ \\
\hline & & $\sigma$ & $0.183(8)$ & $0.227(9)$ & $0.247(12)$ & $0.291(16)$ \\
\hline
\end{tabular}

$\left(\mathrm{HNO}_{3}\right)+2 \mathrm{ml}$ hydrogen peroxide $\left(\mathrm{H}_{2} \mathrm{O}_{2}\right)$. These concentrations were used to normalize all our magnetic measurements in order to obtain the NP magnetization.

A Levenberg-Marquardt algorithm has been used for all the fittings.

\section{Theory}

If all NPs are in $\tau_{x p}>\tau_{N}$ state and if their anisotropy energy can be neglected, the Langevin function $L(\xi)$ can be used and the dependence of the sample global magnetization on the magnetic induction (MB curves) is given by [32-34]

$$
M(B)=\frac{1}{\langle V\rangle} \int_{0}^{\infty} \mu L\left(\frac{\mu B}{k_{B} T}\right) f_{\log }(r) d r
$$

where $\mu=M_{s}(T) V$ is the magnetic moment of one NP, $\langle V\rangle$ is the mean NP volume, and $M_{s}(T)$ is the saturation magnetization at temperature $T$ and is supposed to be independent of the NP radius. The integral sums on all the radii of the size distribution $f_{\log }(r)$.

\section{Results and Discussion}

TEM histograms with the corresponding lognormal distribution fittings are shown in Figure 1 for S2 and S3, and results of the fittings are given for all samples in Table 1. For each sample, median NP radius ranged from around 3.2 to $3.6 \mathrm{~nm}$ (see Table 1). Distributions were relatively polydispersed: for example, NP radius ranged from 1.75 to $5.25 \mathrm{~nm}$ for S2 and from 2 to $6 \mathrm{~nm}$ for S3 (see Figure 1).

MB curves at $315 \mathrm{~K}, 300 \mathrm{~K}, 275 \mathrm{~K}, 200 \mathrm{~K}$, and $100 \mathrm{~K}$ were fitted with equation (7) with the two parameters of lognormal distribution: median radius $r_{0}$ and $\sigma$. Saturation magnetization $M_{s}$ is entirely reached: at $3 \mathrm{~T}$, magnetization is larger than 99\% (see Figure S1 in supplementary materials) of its maximum value for all samples. For the fittings, saturation magnetization was set to the maximum value obtained after diamagnetic correction. For comparison, the same experiments were also fitted with a simple Langevin, considering all NPs have the same radius $r_{0}$. The data and the corresponding fittings are shown in Figure 2 for S4 and in Table 1 for all samples. Table 1 also allows a comparison with the obtained TEM sizes.

Figure 2 shows that lognormal distribution fitting (LDF) works much better than simple Langevin fitting (SLF). Mean 


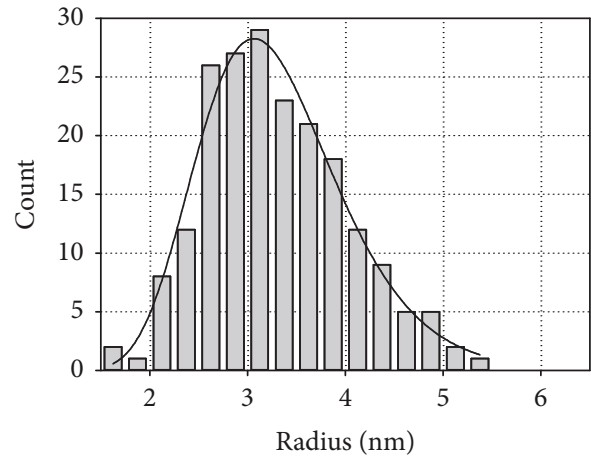

(a)

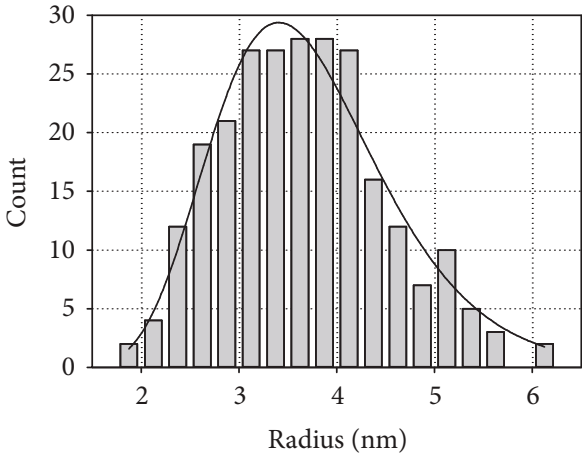

(b)

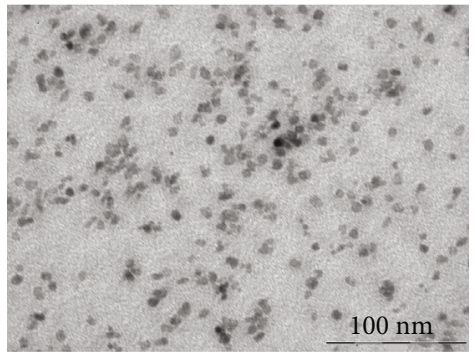

(c)

Figure 1: TEM size histograms with lognormal fittings for (a) S2 and (b) S3. (c) A typical TEM image (S1 in this case).

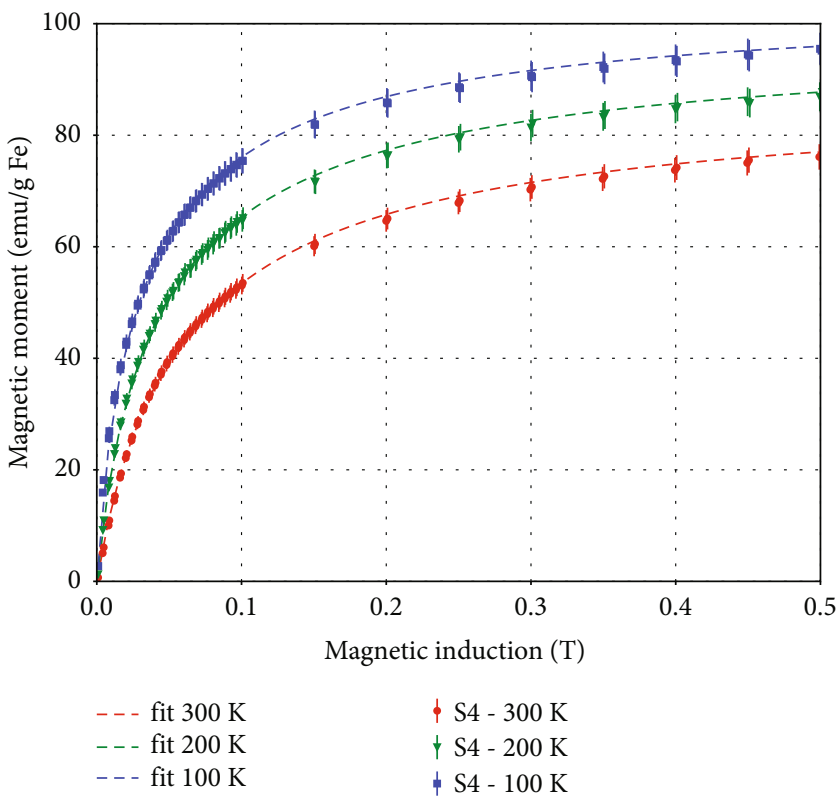

(a)

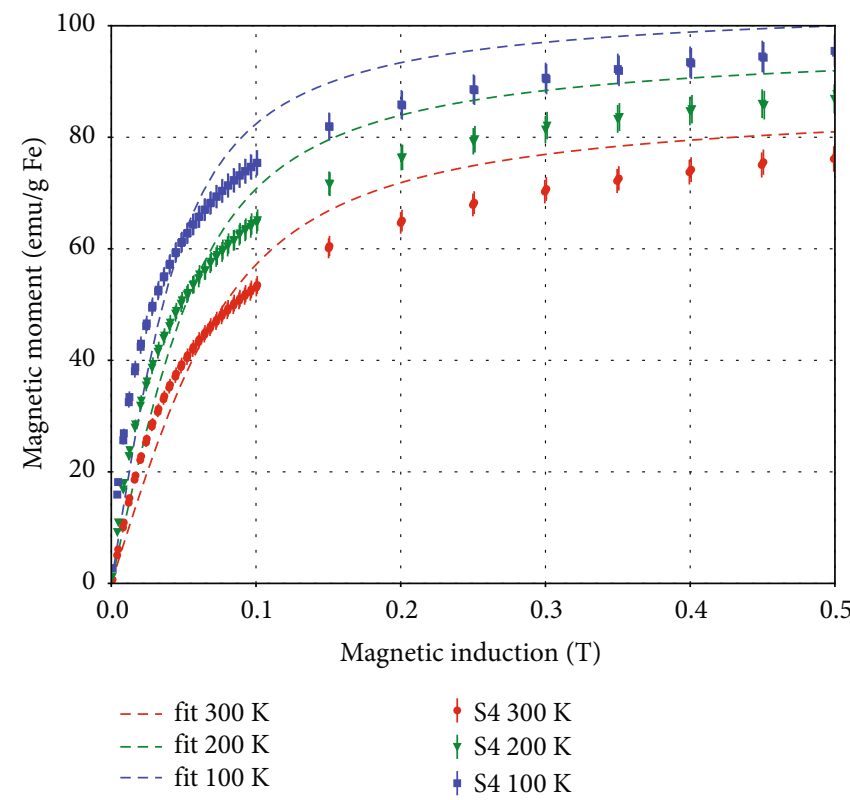

(b)

Figure 2: MB curves from 0 to $0.5 \mathrm{~T}$ for S4 with (a) lognormal distribution fitting and (b) simple Langevin fitting.

adjusted $R_{\text {adj }}^{2}$ for all samples is equal to 0.9998 for LDF while it is equal to 0.9982 for SLF. Furthermore, at $300 \mathrm{~K}$, which is the usual temperature of measurement in the literature [16, $23,25-27], r_{0}$ values for LDF are closer to TEM results than $r_{0}$ obtained for SLF: as expected [20], $r_{0}$ is overestimated for SLF. This result confirms that taking into account the size distribution provides a better estimation of NP size. Com- pared to TEM results, $\mathrm{MB}$ curves at $275 \mathrm{~K}$ and above give globally a better estimation of size distribution.

Table 1 shows that there is a strong influence of temperature on the parameters of LDF: when temperature decreases, median radius $r_{0}$ decreases while $\sigma$ increases. The trend observed for $r_{0}$ is also present but less pronounced with SLF. Of course, this variation has no physical meaning since 


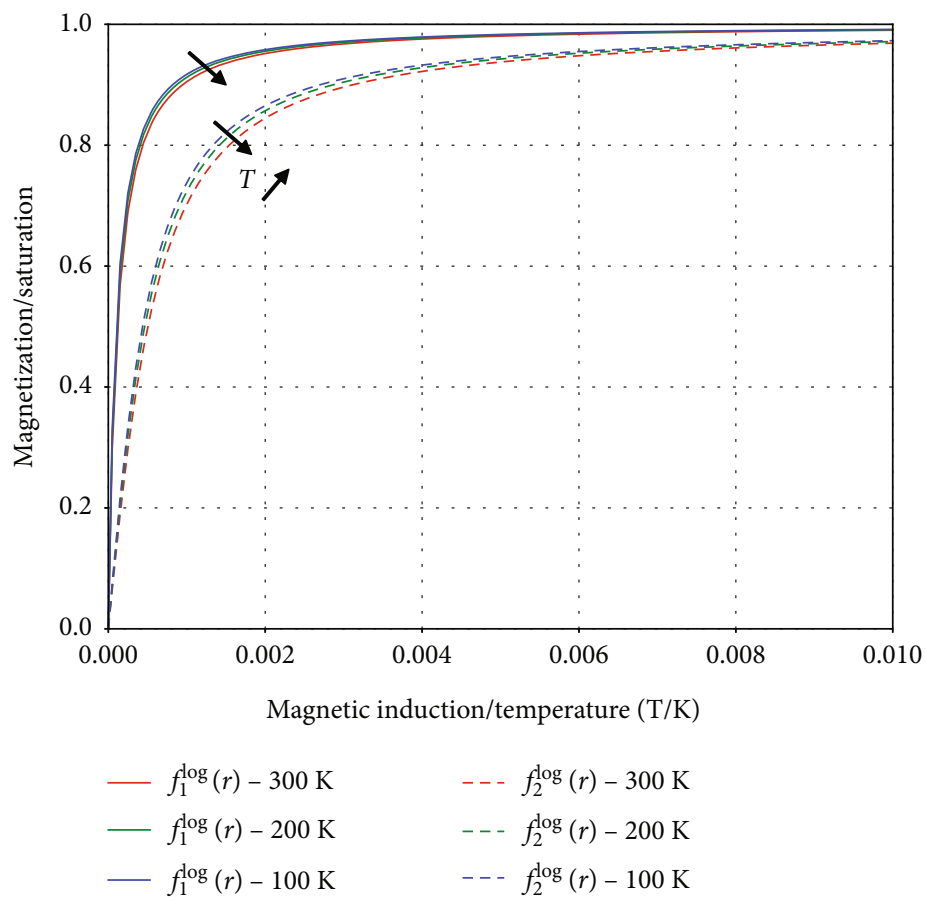

Figure 3: Normalized magnetization $M / M_{s}$ as a function of $B / T$. Theoretical $M B$ curves at $300 \mathrm{~K}, 200 \mathrm{~K}$, and $100 \mathrm{~K}$ for two different distributions $\left(f_{1}^{\log }(r): r_{0}=4 \mathrm{~nm}\right.$ and $\sigma=0.3 ; f_{1}^{\log }(r): r_{0}=3 \mathrm{~nm}$ and $\left.\sigma=0.15\right)$.

the sample remains the same. This unexpected experimental result was never mentioned in literature before. To verify if the Langevin law is suited to describe the experimental data, the test generally used [35] is to plot normalized magnetization as a function of $B / T$, the magnetic induction normalized by temperature.

If the Langevin law is valid, MB curves obtained at different temperatures should superimpose. However, this test was only performed in the literature with monodispersed NPs. Therefore, we first checked numerically that the normalized magnetization of a polydispersed population of NPs following the Langevin law was indeed superposing when plotted as a function of $B / T$, as shown in Figure 3 . The test thus seems to work even for polydispersed samples: for each distribution, $\mathrm{MB}$ curves are very close, even if they are slightly shifted down when temperature increases.

Experimental normalized magnetizations are represented in Figure 4 for S2 and S3. MB curves at different temperatures do not superimpose completely, and they are slightly shifted up when temperature increases, so exactly the opposite of what is expected from the numerical computation (Figure 3). This deviation demonstrates that the Langevin law is not suited anymore when temperature decreases.

This type of deviation was already predicted by Respaud [35] based on the fact that anisotropy energy is not taken into account in the Boltzmann statistics from which the Langevin law is derived. Considering a same size for all NPs, he demonstrated by numerical calculations that anisotropy energy will be responsible of a visible deviation for points with $\xi=$ $\mu B / k_{B} T>1$ as soon as $\lambda=K_{A} V / k_{B} T>2$. That is exactly what is observed in Figure 4: deviation is visible as soon as $\xi>1$ which corresponds to $B / T$ equal to around 0.0002 for $S 1$ and S2, 0.00015 for S3, and 0.0004 for S4. Using TEM results for the radius and standard deviation and $K_{A}=15000 \mathrm{~J} / \mathrm{m}^{3}$ $[10,16-19]$, calculations at $100 \mathrm{~K}$ give $46 \% \mathrm{NPs}$ satisfying $\lambda$ $>2$ criterion for S3 and 35\% for S4. At $300 \mathrm{~K}$, it is only $6 \%$ and $5 \%$, respectively. So, the number of NPs contributing to visible deviation from the Langevin law logically increases as temperature decreases. Zero-Field-Cooling results (not shown here) even suggest that $K_{A}$ for samples studied are rather between 25000 and $35000 \mathrm{~J} / \mathrm{m}^{3}$. With these $K_{A}$ values, NP proportion satisfying $\lambda>2$ criterion at $100 \mathrm{~K}$ would be $80 \%$ for S3 and $75 \%$ for S4. At $300 \mathrm{~K}$, it would be still $25 \%$ and $20 \%$, respectively. These results confirm the predictions of Respaud [35] and the fact that anisotropy energy has to be taken into account in the Boltzmann statistics to obtain a good determination of lognormal size distribution parameters.

It is therefore not surprising that the distribution parameters obtained by fitting MB curves are, compared to TEM results, the best at room temperature and are the worst at $100 \mathrm{~K}$ (see Table 1). Because of anisotropy deviation effect, the Langevin law is less and less relevant as soon as temperature decreases. Because experimental MB curves are slightly shifted up when temperature increases, which does not correspond to what is expected for the Langevin law, LDF which uses the Langevin law gives irrelevant size distribution results to fit to the data. Indeed, when the temperature decreases, the median radius $r_{0}$ is artificially decreased and $\sigma$ is artificially increased to compensate the difference between theory and experiments.

In liquid water, since all the studied NPs have a radius $r_{H}<115 \mathrm{~nm}$, their magnetic moment is not blocked thanks to Brown relaxation (see Section 1.2). It would thus be 


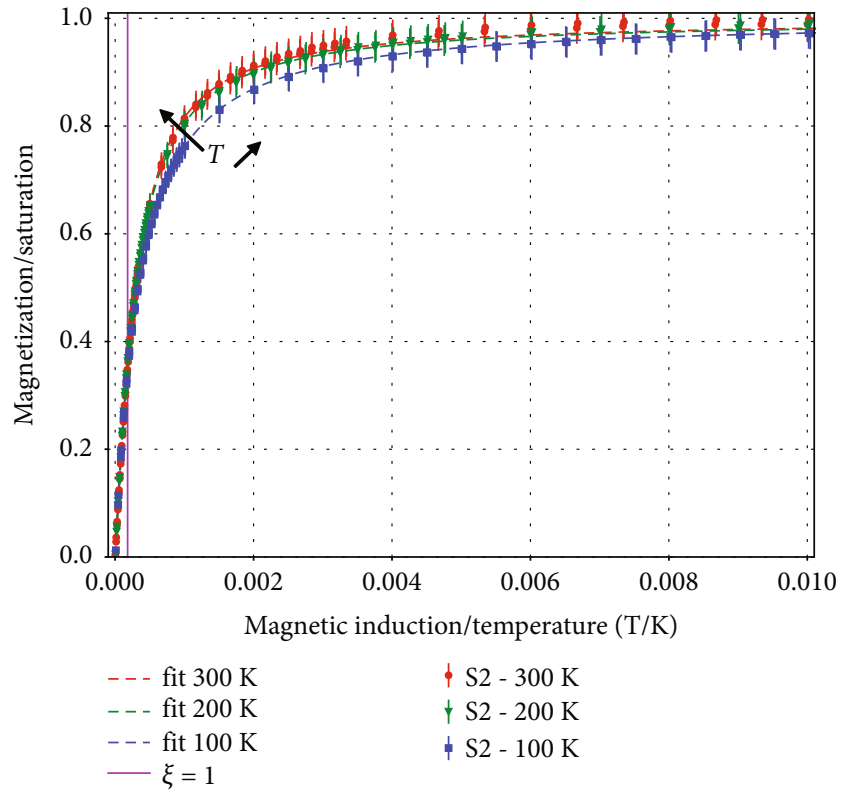

(a)

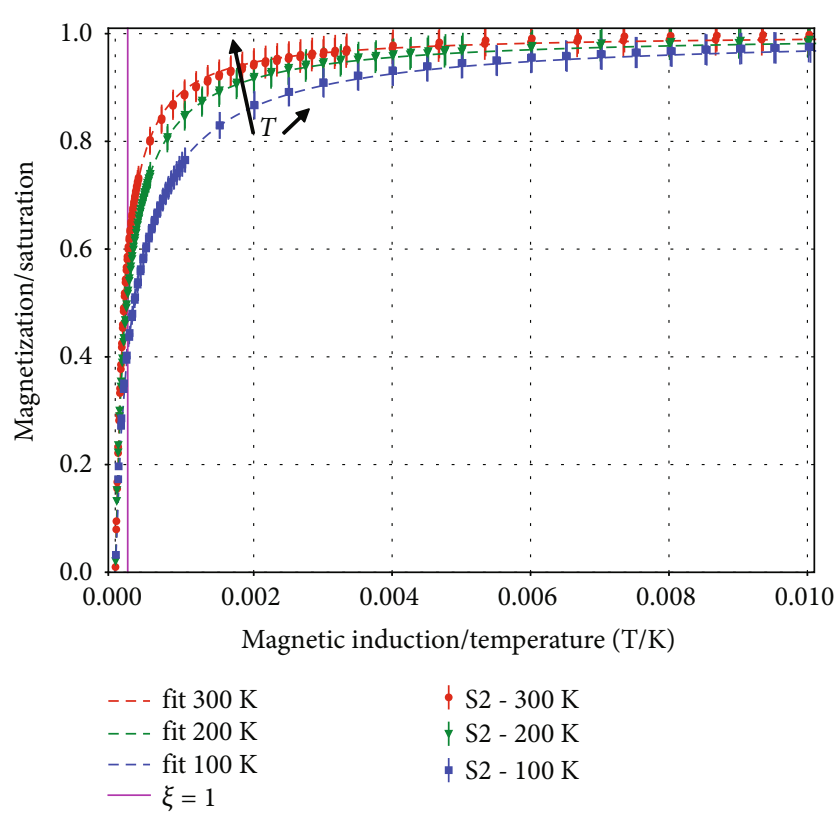

(b)

FIgURE 4: Normalized magnetization curves for (a) S2 and (b) S3 with LDF. The vertical line corresponds to the case $\xi=1$.

expected that, when $T>273.15 \mathrm{~K}$, Brown relaxation would allow anisotropy axis alignment with magnetic induction. In this hypothesis, no temperature deviation would be expected anymore. In fact, a temperature dependence of $r_{0}$ and $\sigma$ parameters is still observed when $T>273.15 \mathrm{~K}$ (see Table 1). This result shows that temperature deviation will occur as soon as anisotropy energy is not taken into account in the Boltzmann statistics, no matter if only Néel relaxation is present or if Brown relaxation also exists. Indeed, Brown relaxation allows to reach global sample magnetization equilibrium faster but this equilibrium is only determined by magnetic, anisotropy, and thermal energies. Thus, only $\lambda>2$ criterion is important to determine if a deviation from the Langevin law will be observed or not. Calculations have shown that even at $300 \mathrm{~K}$, there are still $6 \%$ NPs satisfying $\lambda>2$ criterion for S3 and 5\% for S4 $(25 \%$ and $20 \%$, respectively, according to ZFC values) which explains why there is still an effect of temperature on the values of $r_{0}$ and $\sigma$ obtained by fitting MB curves when $T>273.15 \mathrm{~K}$. In conclusion, care must be taken in future studies: size distribution must be estimated with a $\mathrm{MB}$ curve on a liquid sample at room temperature (or even higher) to minimize the deviation from the Langevin law caused by anisotropy.

\section{Conclusion}

It has been shown that NP size estimation is better with lognormal distribution fitting (LDF) of MB curves than with simple Langevin fitting (SLF) and that LDF is easy to implement. Furthermore, an unexpected result has been observed: when temperature decreases, the obtained $r_{0}$ decreases while $\sigma$ increases. This is due to the fact that the Langevin law is not completely relevant when temperature decreases: anisotropy energy, not taken into account in the Boltzmann statistics, becomes too strong compared to thermal energy. Calculations for our experiments at $100 \mathrm{~K}$ have confirmed that $46 \%$ NPs for S3 and 35\% for S4 contribute to visible deviation from the Langevin law. A smaller temperature dependence has been observed also for temperatures $T>273.15 \mathrm{~K}$ : Brown relaxation does not totally prevent the temperature deviation from the Langevin law. Only $\lambda>2$ criterion plays a role: calculations have shown that even at $300 \mathrm{~K}$, there are still $6 \%$ NPs for S3 and 5\% for S4 that contribute to visible deviation from the Langevin law. Therefore, it is better to estimate NP size distribution with the fitting of $\mathrm{MB}$ curve of a liquid sample at room temperature (or even higher) to minimize deviation anisotropy effect.

To go further, it could be interesting to try to adjust several MB curves at different temperatures, including low temperatures as $100 \mathrm{~K}$, with a model taking into account anisotropy energy in the Boltzmann statistic law. Since the Langevin law is no longer appropriate, numerical calculations would be necessary.

Moreover, comparing the results obtained with $\mathrm{MB}$ curves to those obtained with FC-ZFC curves would also bring additional interesting information even if it can complicate the data interpretation.

Another interesting investigation topic would be to freeze samples under strong magnetic induction. This would favour a magnetization direction which would influence all following measurements.

\section{Data Availability}

All the data are available on demand on my office computer (UMONS). 


\section{Conflicts of Interest}

The authors declare that there is no conflict of interest regarding the publication of this paper.

\section{Acknowledgments}

The authors would like to thank Dr. Simo Spassov for fruitful discussions about the data analysis of magnetometry measurements.

\section{Supplementary Materials}

There is only one figure to show that saturation is well reached from 3T. (Supplementary Materials)

\section{References}

[1] L. Blaney, "Magnetite $\left(\mathrm{Fe}_{3} \mathrm{O}_{4}\right)$ : properties, synthesis, and applications," Lehigh Review, vol. 15, no. 5, pp. 32-81, 2007.

[2] Y.-X. J. Wang, S. M. Hussain, and G. P. Krestin, "Superparamagnetic iron oxide contrast agents: physicochemical characteristics and applications in $\mathrm{mr}$ imaging," European Radiology, vol. 11, no. 11, pp. 2319-2331, 2001.

[3] C. Westbrook, C. Kaut Roth, and J. Talbot, MRI in Practice, Wiley-Blackwell, 4th edition, 2012.

[4] Q. L. Vuong, J.-F. Berret, J. Fresnais, Y. Gossuin, and O. Sandre, "A universal scaling law to predict the efficiency of magnetic nanoparticles as MRI T2-contrast agents," Advanced Healthcare Materials, vol. 1, no. 4, pp. 502-512, 2012.

[5] A. Glaria, S. Soulé, N. Hallali et al., "Silica coated iron nanoparticles: synthesis, interface control, magnetic and hyperthermia properties," RSC Advances, vol. 8, pp. 32146-32156, 2018.

[6] P. de la Presa, N. Mille, J. Carrey et al., "Hybrid nanoparticles for magnetic and plasmonic hyperthermia," Physical Chemistry Chemical Physics, vol. 20, no. 37, pp. 24065-24073, 2018.

[7] A. Riedinger, P. Guardia, A. Curcio et al., "Subnanometer local temperature probing and remotely controlled drug release based on azo-functionalized iron oxide nanoparticles," Nano Letters, vol. 13, no. 6, pp. 2399-2406, 2013.

[8] P. Clerc, P. Jeanjean, N. Hallali et al., "Targeted magnetic intralysosomal hyperthermia produces lysosomal reactive oxygen species and causes caspase-1 dependent cell death," Journal of Controlled Release, vol. 270, pp. 120-134, 2018.

[9] H. Kakwere, M. P. Leal, M. E. Materia et al., "Functionalization of strongly interacting magnetic nanocubes with (thermo)responsive coating and their application in hyperthermia and heat-triggered drug delivery," ACS Applied Materials \& Interfaces, vol. 7, no. 19, pp. 10132-10145, 2015.

[10] P. Guardia, B. Batlle-Brugal, A. G. Roca et al., "Surfactant effects in magnetite nanoparticles of controlled size," Journal of Magnetism and Magnetic Materials, vol. 316, no. 2, pp. e756-e759, 2007.

[11] J. Fresnais, C. Wilhelm, G. Nebewia, E. Cazares Cortes, M. Nerantzaki, and C. Ménager, "Magnetic nanoparticles create hot spots in polymer matrix for controlled drug release," Nanomaterials, vol. 8, no. 10, p. 850, 2018.

[12] R. E. Rosensweig, "Heating magnetic fluid with alternating magnetic field," Journal of Magnetism and Magnetic Materials, vol. 252, pp. 370-374, 2002.
[13] J. Carrey, B. Mehdaoui, and M. Respaud, "Simple models for dynamic hysteresis loop calculations of magnetic singledomain nanoparticles: application to magnetic hyperthermia optimization," Journal of Applied Physics, vol. 109, no. 8, article 083921, 2011.

[14] A. K. A. Silva, C. Ménager, and C. Wilhelm, "Magnetic drug carriers: bright insights from light-responsive magnetic liposomes," Nanomedicine, vol. 10, no. 18, pp. 2797-2799, 2015.

[15] F. Gazeau, K. Aubertin, S. Bonneau et al., "Combining magnetic nanoparticles with cell derived microvesicles for drug loading and targeting," Nanomedicine: Nanotechnology, Biology and Medicine, vol. 11, no. 3, pp. 645-655, 2015.

[16] M. Lévy, C. Wilhelm, M. Devaud, P. Levitz, and F. Gazeau, "How cellular processing of superparamagnetic nanoparticles affects their magnetic behavior and NMR relaxivity," Contrast Media \& Molecular Imaging, vol. 7, no. 4, pp. 373-383, 2012.

[17] M. Pauly, B. P. Pichon, P. Panissod et al., "Size dependent dipolar interactions in iron oxide nanoparticle monolayer and multilayer langmuir-blodgett films," Journal of Materials Chemistry, vol. 22, no. 13, article 6343, 2012.

[18] M. Basini, T. Orlando, P. Arosio et al., "Local spin dynamics of iron oxide magnetic nanoparticles dispersed in different solvents with variable size and shape: ${ }^{1} \mathrm{H}$ NMR study," The Journal of Chemical Physics, vol. 146, no. 3, article 034703, 2017.

[19] D. Fiorani, A. M. Testa, F. Lucari, F. D’Orazio, and H. Romero, "Magnetic properties of maghemite nanoparticle systems: surface anisotropy and interparticle interaction effects," Physica B: Condensed Matter, vol. 320, no. 1-4, pp. 122-126, 2002.

[20] A. Roch, R. N. Muller, and P. Gillis, "Water relaxation by spm particles: neglecting the magnetic anisotropy? A caveat," Journal of Magnetic Resonance Imaging, vol. 14, no. 1, pp. 94-96, 2001.

[21] R. Chantrell, J. Popplewell, and S. Charles, "Measurements of particle size distribution parameters in ferrofluids," IEEE Transactions on Magnetics, vol. 14, no. 5, pp. 975-977, 1978.

[22] P. P. Vaishnava, U. Senaratne, E. C. Buc et al., "Magnetic properties of $\gamma-\mathrm{Fe}_{2} \mathrm{O}_{3}$ nanoparticles incorporated in a polystyrene resin matrix," Physical Review B, vol. 76, no. 2, article 024413, 2007.

[23] G. F. Goya, T. S. Berquó, and F. C. Fonseca, "Static and dynamic magnetic properties of spherical magnetite nanoparticles," Journal of Applied Physics, vol. 94, no. 5, article 3520, 2003.

[24] K. Nadeem, H. Krenn, T. Traussnig, R. Würschum, D. V. Szabó, and I. Letofsky-Papst, "Spin-glass freezing of maghemite nanoparticles prepared by microwave plasma synthesis," Journal of Applied Physics, vol. 111, no. 11, article 113911, 2012.

[25] M. Lévy, F. Gazeau, J.-C. Bacri, C. Wilhelm, and M. Devaud, "Modeling magnetic nanoparticle dipole-dipole interactions inside living cells," Physical Review B, vol. 84, no. 7, article 075480, 2011.

[26] J. A. Ramos Guivar, A. Bustamante, J. Flores et al., "Mössbauer study of intermediate superparamagnetic relaxation of maghemite $\left(\gamma-\mathrm{Fe}_{2} \mathrm{O}_{3}\right)$ nanoparticles," Hyperfine Interactions, vol. 224, no. 1-3, pp. 89-97, 2014.

[27] A. Shankar, M. Chand, G. A. Basheed, S. Thakur, and R. P. Pant, "Low temperature fmr investigations on double surfactant water based ferrofluid," Journal of Magnetism and Magnetic Materials, vol. 374, pp. 696-702, 2015. 
[28] K. O’Grady and A. Bradbury, "Particle size analysis in ferrofluids," Journal of Magnetism and Magnetic Materials, vol. 39, no. 1-2, pp. 91-94, 1983.

[29] D. M. Coutinho and V. M. S. Verenkar, "Spin canting and surface spin disorder in ni substituted co-cd ferrite nanoparticles synthesised by fuel deficient combustion method," Journal of Alloys and Compounds, vol. 782, pp. 392-403, 2018.

[30] K. Nadeem, M. Kamran, H. Abbas et al., "Reduced surface spin disorder in $\mathrm{ZrO}_{2}$ coated $\gamma-\mathrm{Fe}_{2} \mathrm{O}_{3}$ nanoparticles," Solid State Communications, vol. 284-286, pp. 69-74, 2018.

[31] M. Vasilakaki, K. Trohidou, A. Douvalis et al., "Assemblymediated interplay of dipolar interactions and surface spin disorder in colloidal maghemite nanoclusters," Nanoscale, vol. 6, no. 7, pp. 3764-3776, 2014.

[32] C. Kittel, Introduction to Solid State Physics, Wiley, Hoboken, NJ, USA, 8th edition, 2012.

[33] C. P. Bean and J. D. Livingston, "Superparamagnetism," Journal of Applied Physics, vol. 30, no. 4, article S120, 1959.

[34] Y. Gossuin, P. Gillis, A. Hocq, Q. L. Vuong, and A. Roch, "Magnetic resonance relaxation properties of superparamagnetic particles," Nanomedicine and Nanobiotechnology, vol. 1, no. 3, pp. 299-310, 2009.

[35] M. Respaud, "Magnetization process of noninteracting ferromagnetic cobalt nanoparticles in the superparamagnetic regime: deviation from langevin law," Journal of Applied Physics, vol. 86, no. 1, pp. 556-561, 1999. 


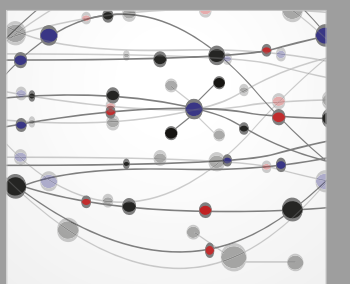

The Scientific World Journal
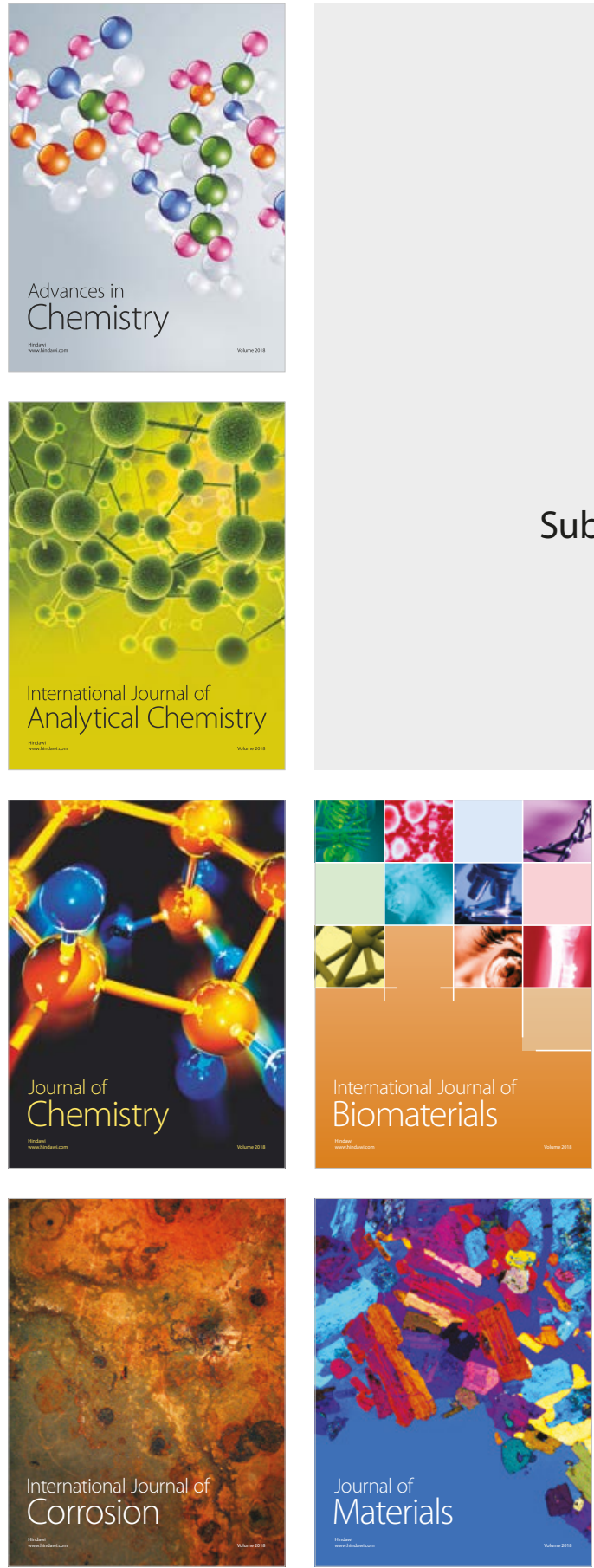

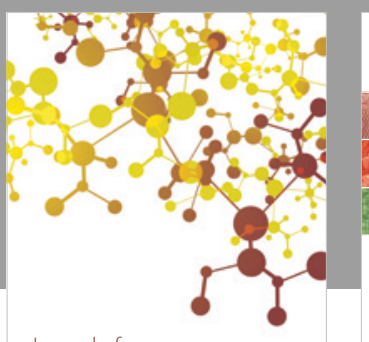

Journal of

Applied Chemistry
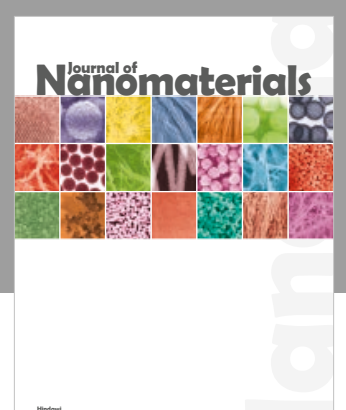

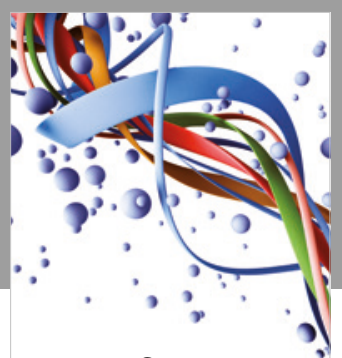

Scientifica

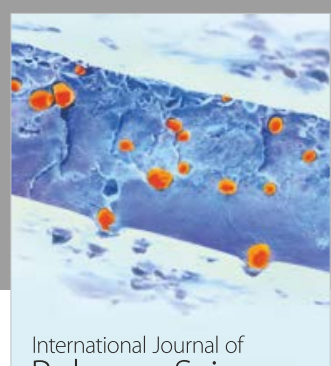

Polymer Science

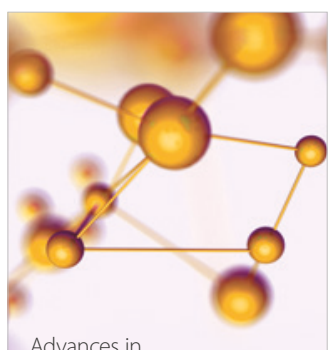

Physical Chemistry
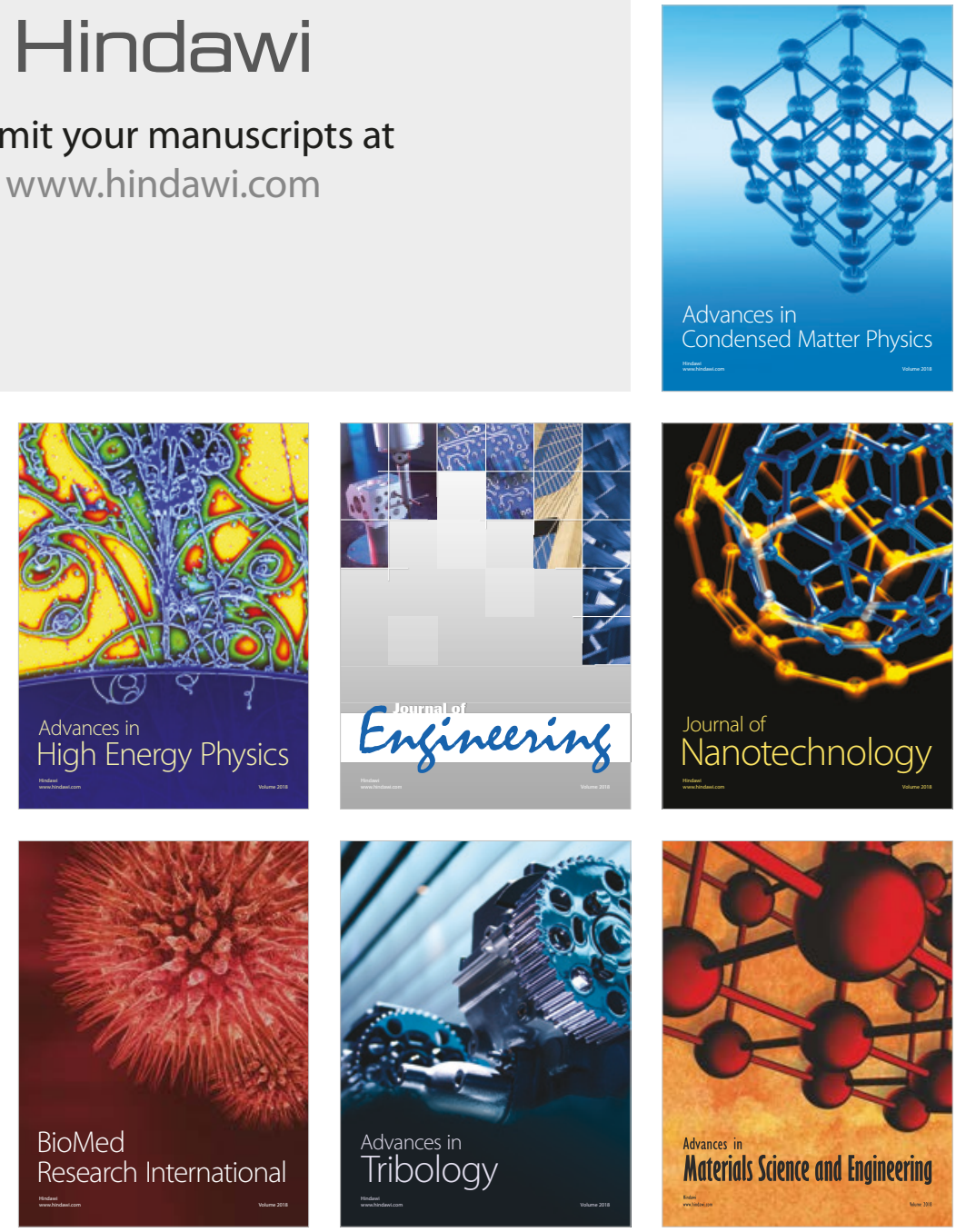